\title{
Functional outcomes after urinary diversion: The importance of long-term assessment
}

\author{
Keith Rourke, MD, FRCSC
}

See related article on page 328.

$\mathrm{R}^{2}$ adical cystectomy (RC) has been used to treat bladder cancer for over 100 years. Over time, therapy has evolved from a strictly ablative approach to one aimed at preserving health-related quality of life (HR-QOL). Orthotopic lower urinary tract (LUT) diversion represents the most recent form of LUT reconstruction after RC. Despite many advances, the optimal form of urinary diversion after cystectomy has yet to be definitively demonstrated. ${ }^{1}$ No randomized controlled trial exists comparing existing forms of urinary diversion and no true consensus has been achieved. This may be related in part to the underlying fact that improved health status does not always translate to a demonstrably better quality of life. ${ }^{2}$ This current article examines both renal and functional outcomes of orthotopic ileal neobladder diversion after RC. ${ }^{3}$ Using a cohort of 31 patients, Lantz and colleagues describe changes in serum creatinine, continence and reservoir emptying 1-year post-continent diversion. A significant rise in serum creatinine from a normal baseline and a $17.9 \%$ rate of uretero-ileal stricture formation were found. Eighty-nine percent of patients developed acceptable daytime continence at 1-year (defined as 0 to 1 pads used per day).

Ultimately, patients with a urinary diversion are at risk of upper urinary tract deterioration. Up to $27 \%$ of patients with ileal conduit diversion develop renal dysfunction that may continue to worsen in a time-dependent manner. These changes may not become obvious for 10 to 20 years after urinary diversion. Detrimental change in renal function may be due to chronic urinary tract infection, ureteral obstruction or high neobladder pressures. This current study demonstrates a $26.3 \mu \mathrm{mol} / \mathrm{L}$ increase in serum creatinine from baseline 1-year after neobladder creation. A significant change occurred with or without the presence of hydronephrosis. The implications of these early findings highlight the need for meticulous long-term assessment of renal function and routine imaging of the upper urinary tract after urinary diversion.

In this study, assessment of continence at 1 -year revealed daytime and nighttime continence rates of $89 \%$ and $70 \%$, respectively. Although no standard definition of continence or voiding function exists for patients after orthotopic diversion, these early results are encouraging and consistent with most published reports. After 12 months, it is thought that sphincteric function and full neobladder compliance have been achieved. However, deterioration in continence may occur gradually 5 years after orthotopic diversion. It is thought that sphincteric function may actually deteriorate with age and time. ${ }^{4}$ This further emphasizes the need for long-term assessment even when evaluating voiding function after continent diversion.

When reporting functional outcomes after LUT reconstruction, it is also imperative to consider HR-QOL. It is widely assumed patients undergoing orthotopic diversion enjoy a better HR-QOL than those undergoing ileal conduit diversion. Currently, there is no definitive evidence. ${ }^{1}$ Studying HR-QOL measures in a prospective manner will help determine the ideal form of urinary diversion and further optimize patient outcomes. Implementation of HR-QOL outcomes specific to urology will likely play a large role in the future assessment of all forms of LUTreconstruction.

Ultimately, long-term outcomes after orthotopic continent diversion are important to document any potential adverse change in renal function or urinary continence. If long-term continence is not achieved or is achieved at the expense of renal function, orthotopic diversion may not actually preserve HR-QOL. The ideal form of LUT replacement is still unknown, but with prolonged follow-up the ileal neobladder seems most likely to emerge as the gold standard of urinary diversion after RC.

Associate Professor, Division of Urology, Department of Surgery, University of Alberta, Edmonton, AB

Competing interests: None declared.

This paper has been peer-reviewed.

\section{References}

1. Gerharz EW, Mansson A, Hunt S, et al. Quality of life after cystectomy and urinary diversion: An evidence based analysis. J Urol 2005;174:1729-36.

2. Lee CT. Quality of life following incontinent cutaneous and orthotopic urinary diversions. Curr Treat Options Oncol 2009; 10:275-86.

3. Lantz AG, Saltel ME, Cagiannos I. Renal and functional outcomes following cystectomy and neobladder reconstruction. Can Urol Assoc J 2010;4:328-31.

4. Madersbacher S, Möhrle K, Burkhard F, et al. Long-term voiding pattern of patients with ileal orthotopic bladder substitutes. J Urol 2002;167:2052-7.

Correspondence: Dr. Keith Rourke, Alberta Urology Institute, Suite 400 Hys Centre, 11010-101 St., Edmonton, AB T5H 4B9; krourke@aburologyinstitute.com 